\title{
Médiévales
}

Langues, Textes, Histoire

50 | printemps 2006

Sociétés nordiques en politique ( $\mathrm{XII}{ }^{\mathrm{e}}-\mathrm{XV}^{\mathrm{e}}$ siècles)

\section{Légitimer l'intégration à l'Occident chrétien : la politique matrimoniale des rois de Norvège au XIII siècle}

How to legitimate the integration into the Western Christendom : the wedding policy of the Kings of Norway in the thirteenth century

\section{Florent Lenègre}

\section{(Q) OpenEdition}

\section{Journals}

Édition électronique

URL : https://journals.openedition.org/medievales/1349

DOI : 10.4000/medievales. 1349

ISSN : $1777-5892$

Éditeur

Presses universitaires de Vincennes

Édition imprimée

Date de publication : 1 juin 2006

Pagination : $59-76$

ISBN : 2-84292-186-0

ISSN : 0751-2708

\section{Référence électronique}

Florent Lenègre, «Légitimer l'intégration à l'Occident chrétien : la politique matrimoniale des rois de Norvège au xIII siècle », Médiévales [En ligne], 50 I printemps 2006, mis en ligne le 15 septembre 2008, consulté le 24 avril 2022. URL : http://journals.openedition.org/medievales/1349 ; DOI : https:// doi.org/10.4000/medievales.1349

Ce document a été généré automatiquement le 24 avril 2022.

Tous droits réservés 


\title{
Légitimer l'intégration à l'Occident chrétien : la politique matrimoniale des rois de Norvège au XIII ${ }^{\mathrm{e}}$ siècle
}

\author{
How to legitimate the integration into the Western Christendom : the wedding \\ policy of the Kings of Norway in the thirteenth century
}

Florent Lenègre

1 À partir de la fin du xI siècle, un Occident chrétien commença à s'affirmer sur les cendres de l'empire carolingien. Les différents royaumes qui le composaient reprirent petit à petit conscience des valeurs qu'ils avaient en commun : une religion, la religion chrétienne, une langue, le latin, et une histoire commune, marquée particulièrement par l'empire romain d'Occident et l'empire carolingien. La papauté joua un rôle central dans ce processus : l'unité des princes chrétiens était une condition nécessaire pour une propagation efficace de la foi chrétienne.

2 Pour promouvoir la bonne entente entre les souverains, la papauté travailla surtout à réglementer leurs usages matrimoniaux. Le mariage était un terrain particulièrement sensible : à la fois source d'alliances et de discordes, il permettait l'accroissement des patrimoines et la production d'héritiers. En cela, il constituait bien l'élément de base de toute politique dynastique. Les mariages multiples, conclus au gré d'alliances changeantes, ainsi que la participation aux successions des enfants naturels étaient une grande source de conflits au sein de l'aristocratie. Au début du xiII ${ }^{\mathrm{e}}$ siècle, lors du concile de Latran IV (1215), la papauté parvint à imposer définitivement le mariage unique et indissoluble, assorti de règles de succession nettes donnant l'exclusivité aux enfants légitimes.

Ce mariage unique, en jouant sur l'importance des solidarités familiales, devait aider à garantir la paix et la cohésion dans l'Occident chrétien. Les grandes familles occidentales continuèrent à se marier entre elles mais établirent des liens familiaux plus stables qui couvrirent bientôt l'Occident entier. Apportèrent-ils la cohésion et la sérénité escomptées? Le traité de Paris en 1259, qui restaurait de bonnes relations 
entre la France et l'Angleterre, fut par exemple conclu entre deux beaux-frères, Louis IX et Henri III, et garanti par plusieurs mariages. Le mariage semble bien être un moyen de réconciliation et de cohésion durable pour les grands souverains occidentaux. Ce phénomène s'étendit-il aux royaumes d'Europe du Nord ou de l'Est récemment convertis et autrefois en conflit avec l'Occident? Parvinrent-ils grâce à une politique matrimoniale adaptée à normaliser leurs relations avec l'Occident voire à s'y intégrer ? L'exemple du royaume de Norvège au xIII $^{\mathrm{e}}$ siècle est dans cette perspective particulièrement éclairant.

4 Lorsqu'on parle de la politique matrimoniale des rois de Norvège au XIII ${ }^{\mathrm{e}}$ siècle, le premier événement qui vient à l'esprit est l'épisode tristement célèbre du mariage, prévu pour la fin de l'année 1290, de la jeune Marguerite de Norvège avec Édouard de Caernavon. La première était la fille unique du roi de Norvège Erik Magnusson, née en 1283. Par sa mère, Marguerite d'Écosse, elle se trouvait être également la petite-fille du roi d'Écosse Alexandre III. À la mort de sa mère en 1283 et de ses deux oncles, disparus sans héritiers en 1281 et 1284, elle se trouva être l'unique héritière du trône d'Écosse et fut reconnue comme telle lors de l'assemblée de Scone en février 1284. Lorsque Alexandre III mourut prématurément d'un accident de cheval en mars 1286 sans avoir laissé d'autres descendants, elle devenait légitimement reine d'Écosse. Édouard de Caernavon n'était rien moins de son côté que le premier prince de Galles, le fils aîné du roi d'Angleterre Édouard I Ir, le futur Édouard II.

5 Si ce mariage entre ces deux jeunes gens est tristement célèbre, c'est parce qu'il n'a jamais été célébré : à peine Marguerite était-elle arrivée dans les Orcades pour conclure cette affaire qu'elle mourait dans des circonstances obscures à la fin du mois de septembre $1290^{1}$. Là se perdait une chance unique d'unir les couronnes d'Angleterre, d'Écosse et de Norvège et peut-être de recréer un empire similaire à celui de Knut le Grand, un empire maritime, typique de cette aire géographique centrée sur la mer du Nord, dont le contrôle et l'unité impliquent la maîtrise de la mer et qui en cela diffère de l'empire terrestre de tradition carolingienne. Par ailleurs, ce mariage aurait également peut-être permis d'éviter le conflit qui allait suivre entre l'Angleterre et l'Écosse à partir de 1296.

Or ce mariage n'était pas, comme on le croit souvent, une affaire strictement angloécossaise. Il fut le résultat d'un demi-siècle d'actions diplomatiques norvégiennes pour s'allier par mariage aux monarchies des îles Britanniques et, en particulier, avec le royaume d'Angleterre. Cet effort s'inscrit dans une véritable politique des rois de Norvège qui, depuis les années 1240, sous l'impulsion du roi Håkon Håkonsson (1217-1263), se tournèrent vers l'Occident et cherchèrent à s'y intégrer durablement ${ }^{2}$.

7 Pour mieux comprendre, remontons un peu dans le temps: la Norvège a eu des contacts avec l'Occident depuis les $\mathrm{VI}^{\mathrm{e}}$ et $\mathrm{VII}^{\mathrm{e}}$ siècles, au moins sur le plan commercial ${ }^{3}$. L'âge viking constitua une étape importante : il fut certes une période de conflits, mais aussi de fréquentation intense par les hommes du Nord des royaumes occidentaux, par le biais de la colonisation par exemple. Bien vite, la chrétienté, lasse des raids vikings, entreprit de les pacifier et le meilleur moyen pour qu'ils arrêtassent d'attaquer les chrétiens était de les rendre chrétiens eux-mêmes. Mais la conversion de la Norvège, acquise au milieu du $\mathrm{XI}^{\mathrm{e}}$ siècle, lui suffisait-elle pour s'intégrer pleinement à l'espace politique occidental? Il ne semble pas. Il fallait, pour acquérir une pleine légitimité au sein de cet ensemble, présenter quelque intérêt, économique ou stratégique, et surtout exprimer la volonté d'adopter les normes qui régissaient le fonctionnement des 
monarchies et des sociétés politiques de l'Occident. Le souvenir des Vikings n'était en effet jamais lointain : les princes norvégiens devaient donc tenter de faire disparaître le préjugé de barbarie et d'archaïsme attaché au souvenir de leurs ancêtres et paraître modernes, politiquement et culturellement ${ }^{4}$, en suivant la voie tracée par les royaumes de France et d'Angleterre.

Parmi les "critères d'adhésion", un des plus importants était d'entrer dans la grande famille que formaient à l'époque les souverains occidentaux. La reconnaissance de l'aptitude de la Norvège à participer à une alliance matrimoniale princière fit évidemment l'objet de manœuvres, de négociations. Ce sont ces tractations, qui se poursuivirent tout au long de la deuxième moitié du xiII ${ }^{e}$ siècle, que nous voulons présenter afin de voir si les rois de Norvège parvinrent effectivement à réaliser leurs ambitions. Le cas échéant, il faudra se demander quels moyens ils employèrent et quels bénéfices ils en tirèrent réellement: autrement dit, nous tenterons de juger de l'efficacité, en matière d'intégration, de l'entrée des rois de Norvège dans le réseau familial des rois d'Occident ${ }^{5}$ et nous nous demanderons s'ils poursuivirent ce même objectif tout au long de la période considérée. Trois temps dans cet effort peuvent être dégagés : à partir des années 1240 , le temps de la reconnaissance par la communauté occidentale et des premières approches matrimoniales, puis ce que nous appellerons le temps des réussites discrètes, dans les années 1260-1280, qui déboucha sur un projet apparemment grandiose, mais qui en réalité prit la forme d'un chant du cygne: le mariage entre Marguerite de Norvège et Édouard de Caernavon.

La reconnaissance du roi de Norvège par l'Occident

9 Ce fut principalement l'œuvre du roi Håkon Håkonsson qui ne ménagea pas ses efforts pour développer le rayonnement de la Norvège sur le plan international. Sa première tâche fut de restaurer une autorité royale unique dans son royaume. Ce n'était pas une mince affaire quand on sait que la Norvège était, depuis son unification à la fin du $\mathrm{IX}^{\mathrm{e}}$ siècle, déchirée par des guerres civiles endémiques. Placé sur le trône par le jarl ${ }^{6} \mathrm{Skuli}$, qui comptait jouer le rôle de maire du palais, Håkon dut d'abord lutter contre le parti de Skuli pour s'imposer comme seul pouvoir à la tête de la Norvège, condition sine qua non d'une présence crédible sur le plan international ${ }^{7}$. Il s'attacha ensuite à moderniser son royaume sur le modèle des grandes monarchies occidentales, œuvre qui sera parachevée essentiellement par son fils Magnus ${ }^{8}$. Il restaura la puissance navale du royaume : vers l'est, il affermit sa place dans l'ensemble baltique face aux Danois et aux Suédois et aux gens de Novgorod; vers l'ouest, il œuvra pour réintégrer dans le giron norvégien toutes les anciennes colonies de l'Atlantique Nord, de l'Irlande à l'Islande 9 . Dans les années 1240, il apparaissait donc comme un interlocuteur respecté pour son poids stratégique en mer du Nord et sa flotte inspirait en Occident des craintes sans doute fondées mais peut-être démultipliées par le souvenir des raids vikings.

Incontesté dans son royaume, Håkon eut cependant du mal à faire oublier à la papauté qu'il était de naissance illégitime ${ }^{10}$ : selon les normes posées par Latran IV, il ne pouvait pas être légitimement roi et n'était par conséquent pas reconnu comme tel par le reste de l'Occident. De plus, à un moment où les souverains occidentaux choisissaient leurs épouses dans les familles régnantes des autres royaumes, il était marié avec la fille du jarl Skuli, l'aristocrate norvégien qui l'avait porté au trône, ce qui ne le plaçait pas en termes de prestige sur un pied d'égalité avec les autres rois. Håkon, pour conforter son potentiel militaire et politique, devait respecter les règles dictées par 
Rome : il imposa en 1260 une loi de succession qui conforta le principe d'une monarchie héréditaire.

11 Ce fut vers la papauté que ses efforts se déployèrent surtout: le pape, qui faisait obstacle à sa reconnaissance en vertu des principes canoniques, pouvait seul fournir la dispense qui permettrait à Håkon d'obtenir un couronnement officiel. Håkon était en bonne position pour négocier: à la suite de la perte de la Terre sainte, les papes cherchaient à relancer la croisade, et les Mongols commençaient à déferler sur l'Europe de l'Est. La puissance navale de la Norvège était reconnue à l'époque, et peut-être même légèrement surévaluée comme on l'a dit. Sa position géographique en faisait d'autre part pour la papauté un allié intéressant dans la lutte sans merci qu'elle livrait avec l'empereur romain germanique. Le roi sut faire vibrer cette corde sensible : entre 1241 et 1246 , il multiplia les initiatives auprès du pape, se proposant de lutter contre l'avancée des Mongols et d'organiser une croisade contre ses voisins païens, les Sames et les Finnois du nord de la péninsule scandinave ${ }^{11}$.

Ces efforts d'adaptation furent récompensés en 1247: après plusieurs années de demandes sans résultats concrets, le pape Innocent IV envoya un légat, le cardinal Guillaume de Sabine, pour couronner officiellement Håkon à Bergen, le 29 juillet ${ }^{12}$, contre une promesse de croisade, en Terre sainte cette fois-ci. Cette reconnaissance de la papauté légitimait celle dont il bénéficiait déjà grâce à son importance politicostratégique sur l'échiquier occidental. Håkon se tourna alors résolument vers l'Ouest et les souverains occidentaux ne tardèrent pas à nouer des contacts plus réguliers avec lui, souvent avec quelques arrières-pensées : on ne s'étonnera pas, par exemple, de voir l'empereur Frédéric II se manifester juste après le couronnement pour contrebalancer l'influence du pape avec lequel il était en conflit. Le roi de France Louis IX lui proposa de participer à sa première croisade mais Håkon refusa. Henri III d'Angleterre de son côté, avec lequel il avait noué des contacts commerciaux et diplomatiques dès les années $1220{ }^{13}$, continua à entretenir des relations suivies et amicales ${ }^{14}$.

Håkon avait promu le christianisme, il avait modernisé son royaume, il avait été couronné par les plus hautes instances ecclésiastiques, il avait fait reconnaître l'intérêt de son alliance. Les rois de Norvège devaient désormais pérenniser cette reconnaissance. Ils s'y employèrent, entre autres au moyen d'une politique matrimoniale ciblée.

Premières tentatives

14 Le premier essai de Håkon pour réaliser un mariage royal en Occident se fit en direction de la Castille d'Alphonse X le Sage. La Saga de Håkon Håkonsson en donne un récit assez détaillé ${ }^{15}$. Il est difficile de déterminer qui a pris l'initiative de cette alliance, toujours est-il que la Saga mentionne l'envoi vers la Castille par Håkon le Jeune, fils aîné du roi, d'une délégation chargée de cadeaux ${ }^{16}$. Celle-ci partit au cours de l'été 1255 et arriva en Espagne à la fin de l'année. Les ambassadeurs repartirent, accompagnés d'une délégation castillane, à la fin du printemps 1256 et arrivèrent en Norvège au début de l'automne avec une proposition de mariage. Les Castillans demandaient la main de Christine, fille de Håkon, pour un des frères du roi Alphonse X. Le roi les accueillit bien et leur accorda d'hiverner à Tønsberg, le temps qu'il prît une décision ${ }^{17}$.

Les enjeux de ce mariage étaient suffisants pour justifier cet hiver de réflexion, notamment sur le plan de la politique internationale. Nous sommes à la fin de l'année 1256, Alphonse X était à ce moment-là candidat à l'élection impériale qui devait avoir lieu à Francfort, le 13 janvier 1257. Håkon savait qu'Alphonse briguait sérieusement le 
trône impérial : laisser passer l'hiver était une façon d'attendre le résultat de l'élection de Francfort avant de donner une réponse aux ambassadeurs castillans. Alphonse était en effet en concurrence avec Richard de Cornouailles, le frère de Henri III d'Angleterre avec lequel Håkon avait tout intérêt à rester en bons termes, puisque c'était l'Angleterre qui fournissait à la Norvège une grande partie de son approvisionnement en blé, blé dont elle dépendait de plus en plus à mesure que le XIII ${ }^{\mathrm{e}}$ siècle avançait ${ }^{18}$. Il importait aussi au roi de ne pas s'aliéner le nouvel empereur : il cherchait en effet à obtenir le contrôle de la ville marchande impériale de Lübeck qui dominait le commerce du sel indispensable à la conservation du hareng et de la morue, principal produit d'exportation norvégien, et qui fournissait un blé bon marché, à un moment où les prix en Angleterre commençaient à monter.

Cette alliance était donc potentiellement intéressante pour Håkon mais il lui fallait impérativement miser sur le bon candidat. Un léger problème survint alors : le 13 janvier 1257, les deux candidats furent élus par leurs partisans respectifs, à la suite d'une querelle. Malgré cela, le roi de Norvège donna son accord à ce mariage. On imagine qu'au printemps 1257, il estima que, malgré cette double élection, le rapport de force était favorable à un Alphonse $\mathrm{X}$ qui disait disposer du soutien des rois de France, d'Aragon, de Navarre, de Bohême, de Hongrie et de Portugal et semblait être le favori du pape ${ }^{19}$. C'est donc le prestige d'Alphonse X qui a sans doute fait pencher la balance en faveur de cette union. Pour le roi de Norvège, celle-ci représentait le sésame qui lui permettait d'intégrer le vaste réseau familial des souverains occidentaux dans lequel Alphonse $\mathrm{X}$ était en effet inséré et qui comprenait toutes les grandes familles régnantes occidentales: les rois de France, d'Angleterre, les Hohenstaufen ou les empereurs de Constantinople, pour ne citer qu'eux. S'allier au Castillan par un mariage, c'était entrer dans leur parenté et donc devenir à terme un membre légitime de la communauté qu'ils formaient. Ainsi, après un voyage raconté avec grand détail dans la Saga d'Håkon, Christine épousa Don Felipe, frère d'Alphonse, le 31 mars 1258.

On le voit, Håkon pouvait avoir besoin d'Alphonse s'il devenait empereur mais pourquoi Alphonse, de son côté, aurait-il eu besoin de Håkon, roi de la lointaine Norvège? Outre le soutien qu'un roi de Norvège était susceptible de lui apporter dans son futur Empire, c'était surtout le vœu de croisade de Håkon qui intéressait Alphonse. Celui-ci avait en effet pour projet de lancer une expédition maritime en Afrique du Nord pour combattre les Maures installés au Maroc. La puissante flotte norvégienne aurait été un soutien appréciable pour cette opération et aurait permis de contrôler les côtes pendant que des troupes au sol envahissaient le Maroc ${ }^{20}$. Dans cette perspective, Alphonse X prit bien soin de faire reconnaître cette expédition contre les Maures comme une croisade, afin que le roi de Norvège puisse remplir sa promesse en servant les intérêts castillans. Ce n'est donc pas un hasard si, en plus de la conclusion du mariage de Christine, on procéda en 1258 à la signature d'un traité d'alliance militaire entre la Castille et la Norvège ${ }^{21}$.

18 Ce mariage illustre le cheminement que l'on a décrit plus haut. Il est, de la part des deux rois, le fruit d'un calcul politique. Si Håkon a pu obtenir ce mariage qui lui permettait de s'allier à une prestigieuse famille occidentale, c'est en raison de son potentiel politique et stratégique - réel ou supposé : soutien à un candidat à l'Empire et flotte à la solide réputation - qui lui a permis selon le système du don et contre-don de réaliser un double bénéfice : faire reconnaître la valeur de son alliance et trouver place 
dans le réseau de parenté d'Alphonse $\mathrm{X}$, et ainsi conforter le processus d'intégration durable de la Norvège en Occident, en cas de maintien de sa dynastie au pouvoir.

Ce mariage paraissait donc être une réussite sur tous les plans tant pour le roi de Norvège que pour le roi de Castille. En réalité ce fut un échec complet: Christine mourut sans enfants en 1262 - de langueur selon la tradition -, la croisade de Håkon ne fut jamais entreprise et Alphonse ne devint pas empereur.

Entre-temps, une deuxième tentative norvégienne eut lieu. Håkon, grisé par son succès provisoire en Castille, s'enhardit et se tourna alors vers l'Angleterre : il demanda la main de Béatrice, fille du roi Henri III, pour son fils aîné Magnus. Cette demande est connue par la réponse de Henri III, datée du 14 mai 1259 : il y mentionnait la requête de Håkon et répondait qu'il lui était impossible d'accorder Béatrice à Magnus, car le sort de celle-ci était prévu par le traité de Paris ${ }^{22}$. Henri était-il mal disposé à l'égard du Norvégien, qui dans le cadre de l'élection impériale soutenait Alphonse X depuis 1258 contre son frère, Richard de Cornouailles ? Peut-être, mais la raison en est avant tout que la Norvège n'était ni assez puissante ni assez intégrée à l'ensemble occidental pour pouvoir rivaliser avec le royaume de France : elle n'avait pas assez à proposer. Henri III promit en revanche de trouver une épouse pour Magnus au sein des grandes familles de son royaume mais cela n'aboutit pas.

21 Dans la stratégie politique de Håkon, la conclusion d'un mariage princier au sein des familles royales d'Occident fonctionne donc comme un échange. Ayant réussi à construire la renommée de son royaume, malgré des ressources somme toute limitées, il pouvait briguer, en échange de son appui, des alliances matrimoniales très ambitieuses, qui renforceraient encore son prestige naissant sur l'échiquier européen. Elles échouèrent toutes deux, la première desservie par un mauvais concours de circonstances, la seconde sans doute par excès de présomption.

Une réussite discrète

Le règne de Magnus Håkonsson (1263-1280), dans tous les domaines, continua et consolida l'œuvre de Håkon. Il se distingua particulièrement par une intense activité législative - d'où son surnom de Lagabøter, le Législateur - qui codifia les évolutions qui avaient débuté lors du règne précédent ${ }^{23}$. À l'extérieur, le roi poursuivit les " négociations d'adhésion " avec l'Occident chrétien, avec la volonté de normaliser les relations par la conclusion de traités. Ainsi, le traité de Perth du 2 juillet 1266 régla le problème des Hébrides qui opposait la Norvège à l'Écosse. Il cédait les Hébrides et l'île de Man, qui appartenaient à la Norvège depuis 1098, à l'Écosse contre 4000 marcs et une rente annuelle perpétuelle de 100 marcs ${ }^{24}$. Un traité de libre commerce, le traité de Winchester, était passé le 21 août 1269 avec l'Angleterre ${ }^{25}$. Cela participait de l'effort de modernisation du royaume lancé par Håkon afin d'effacer les préjugés qui s'attachaient obstinément à l'image qu'avaient de la Norvège les peuples plus méridionaux ${ }^{26}$.

Dans le domaine de la politique matrimoniale, Magnus ${ }^{27}$ continua, comme son père, à regarder vers l'Ouest, mais il lui fallait fixer des objectifs raisonnables. Il abandonna donc la grande politique de Håkon sans pour autant renier la méthode. Plus modestement, il amorça peu de temps avant sa mort, le 9 mai 1280, des négociations avec le royaume d'Écosse pour marier son fils aîné Erik avec Marguerite, la fille du roi Alexandre III. Ce mariage se situait dans le prolongement de la normalisation et de la consolidation des relations avec le royaume d'Écosse consécutives au traité de Perth. 

mal renseignés. Ainsi, il est difficile de dire qui fut à l'initiative de ce mariage. Notons juste que ce traité était très favorable à la Norvège, qui éprouvait des difficultés financières croissantes et souhaitait favoriser les relations commerciales avec l'Écosse ${ }^{28}$; en revanche, les Écossais prirent grand soin de faire garantir sa réalisation par les Norvégiens. La chronique de Lanercost précise que c'était le roi d'Écosse qui avait insisté pour conclure ce mariage, dont le but était de consolider la cession des Hébrides à l'Écosse ${ }^{29}$. Cette assertion semble vraisemblable, même si le chroniqueur commet par ailleurs bien des erreurs dans le traitement de cette affaire. Le traité de mariage (conventio) fut conclu à Roxburgh le 25 juillet $1281^{30}$ : il prévoyait le paiement d'une dot de 14000 marcs ${ }^{31}$ dont la moitié pourrait être constituée de rentes sur des terres écossaises. Le douaire de Marguerite s'élevait à 1400 markebøl. L'accord prévoyait que les enfants engendrés par le couple pourraient hériter naturellement de la couronne norvégienne, mais aussi de l'écossaise en cas d'absence d'héritier légitime. Ce traité, conclu en Écosse, fut ensuite envoyé au jeune roi ${ }^{32}$ Erik et à son conseil pour être ratifié définitivement, en même temps que Marguerite, qui répugnait à ce mariage, si on en croit les chroniques écossaises ${ }^{33}$. Elle fut couronnée reine de Norvège à Bergen début septembre $1281^{34}$.

Malgré ses avantages économiques et politiques évidents, ce mariage ne semblait pas à première vue assurer une meilleure intégration de la Norvège à la chrétienté occidentale : le royaume d'Écosse était davantage rattaché à la zone d'influence de la Norvège - mer du Nord, mer d'Irlande et Atlantique Nord - qu'au centre de la chrétienté occidentale et sa réputation n'était pas meilleure que celle du royaume de Norvège en matière de modernité. Pourtant, il se révélait être une magnifique stratégie d'alliance: Marguerite se trouvait être en effet la fille d'Alexandre III et d'une autre Marguerite, fille de Henri III et sœur d'Édouard I ${ }^{\text {er }}$. L'épouse du roi Erik de Norvège était donc la nièce du grand roi Édouard I $\mathrm{I}^{\mathrm{er}} \mathrm{d}$ 'Angleterre dont le réseau familial couvrait l'Occident entier. Le roi de Norvège et surtout l'enfant qui naîtrait de ce mariage devenaient ainsi parents du puissant roi d'Angleterre et de la plupart des souverains occidentaux. Ce mariage ouvrait également la possibilité de renforcer encore davantage la position de la Norvège sur l'échiquier politique occidental: les perspectives en termes de succession dynastique étaient très intéressantes, grâce à la clause qui prévoyait la possibilité pour les enfants à naître d'hériter à la fois des trônes d'Écosse et de Norvège en cas d'absence d'autres héritiers.

Bien que, au moment où le traité fut conclu, cette éventualité ne fût pas à l'ordre du jour - le roi Alexandre III avait toujours un fils, Alexandre, et, même s'il était veuf, il pouvait encore se remarier et engendrer d'autres héritiers -, ce mariage était donc prometteur du point de vue norvégien: il rattachait la dynastie norvégienne aux grandes familles régnantes de l'Occident, et portait en germe la possibilité de créer un vaste empire maritime centré sur la mer du Nord réunissant les couronnes de Norvège et d'Écosse. Malheureusement, Marguerite mourut le 9 avril 1283, moins de deux ans après son couronnement ${ }^{35}$. Elle laissait cependant une petite fille, Marguerite, âgée de quelques mois, qui allait se révéler de santé fragile. C'était sur elle maintenant que reposait le lien dynastique unissant la Norvège au reste de l'Occident. Or, avec la mort du dernier fils d'Alexandre III en janvier $1284^{36}$, elle devint l'unique héritière du roi d'Écosse. Fille unique d'Erik qui était maintenant veuf, elle était potentiellement aussi l'héritière du trône de Norvège, si le frère d'Erik venait à mourir ${ }^{37}$. En février 1284, elle 
fut reconnue comme héritière d'Écosse par l'assemblée de Scone, à l'initiative d'Alexandre III ${ }^{38}$, malgré l'opposition de certains membres de l'aristocratie.

Un projet grandiose en forme de chant du cygne

Alexandre III, conscient du caractère problématique de sa succession, se remaria avec Yolande de Dreux à l'automne $1285^{39}$. Mais, le 19 mars 1286, il mourut d'un accident de cheval sans laisser d'héritier ${ }^{40}$. En vertu de la règle de succession et de la reconnaissance de 1284, c'était donc Marguerite qui devenait reine d'Écosse. La politique matrimoniale des rois de Norvège avait fini par porter ses fruits, de manière finalement assez accidentelle : avec Marguerite sur le trône, ils étaient à même de venir occuper une place de premier plan dans le concert des grandes monarchies occidentales.

Cependant, l'opposition d'une partie de l'aristocratie écossaise, peu encline à accepter une enfant de santé fragile et étrangère sur le trône d'Écosse, se renforçait. Les gardiens nommés pour assurer la régence envoyèrent alors une délégation à Édouard $\mathrm{I}^{\mathrm{er}}$, qui se trouvait en Gascogne, pour l'informer de la situation et peut-être lui demander de l'aide ${ }^{41}$. Les Norvégiens de leur côté étaient bien décidés à faire reconnaître les droits de Marguerite sur le trône d'Écosse. Le baron Bjarne Erlingsson de Bjarkøy, un des plus grands personnages du royaume, fut envoyé en Écosse à l'hiver 1286 , sans doute pour demander cette reconnaissance ${ }^{42}$. Le caractère chaotique de la situation dut le persuader de l'utilité du recours à un médiateur pour parvenir à ce but et pacifier l'Écosse. C'était donc le moment pour Erik d'exploiter les potentialités du traité de 1281 et de faire jouer la parenté qui existait entre Édouard $\mathrm{I}^{\mathrm{er}}$ et Marguerite.

Le $1^{\text {er }}$ avril 1289, il adressait une lettre au roi d'Angleterre pour lui annoncer l'arrivée d'une délégation, «[...] afin de traiter et de discuter avec votre sérénité de certaines affaires nous concernant, nous et notre très chère fille, Marguerite, votre parente, seigneur et reine d'Écosse, et son royaume d'Écosse ${ }^{43}$ ». Par le vocabulaire employé, et notamment celui de consanguinea, on voit qu'Erik cherchait à mettre en valeur les liens de parenté unissant Édouard et Marguerite, de façon à faire jouer la solidarité qui doit exister entre deux membres d'une même famille. De même, Erik s'adressait dans cette lettre à Édouard en ces termes : «Au prince excellent et magnifique, son affin et son ami, messire Édouard ${ }^{44}$. » Il insistait bien sur le fait qu'en vertu du mariage de 1281, ils étaient affines, parents par alliance, ce qui devait inciter Édouard à agir en sa faveur. Édouard $\mathrm{I}^{\mathrm{er}}$ prit un peu de temps pour évaluer la situation puis, dès son retour de Gascogne en 1289, il s'attacha à réunir toutes les parties concernées pour discuter du sort de Marguerite et son éventuelle arrivée en Écosse. Édouard $\mathrm{I}^{\mathrm{er}}$, en cherchant à faire reconnaître Marguerite comme reine d'Écosse, semblait embrasser les intérêts norvégiens. Mais il avait évidemment une idée en tête: obtenir le mariage entre Marguerite, reconnue reine d'Écosse, et son fils aîné Édouard de Caernavon. Son premier objectif dans cette affaire était de pacifier l'Écosse et d'obtenir un droit de regard sur les affaires écossaises. Dans cette perspective, le mariage projeté constituait une bonne occasion. Son deuxième objectif était sans doute d'éviter que l'Écosse et par ricochet la Norvège ne conclussent une alliance matrimoniale avec des ennemis du roi d'Angleterre, à une époque où un conflit se profilait entre les royaumes de France et d'Angleterre et où chaque camp cherchait à construire un réseau d'alliances.

31 Il est important d'observer ici que ce sont les connexions familiales tissées par le traité de 1281 qui ont permis à la famille de Norvège d'acquérir une importance politique suffisante pour entrer dans les plans du roi d'Angleterre. On peut ainsi mesurer tous les 
progrès réalisés en quelque trente ans par le royaume de Norvège sur la voie de l'intégration à l'Occident : l'alliance matrimoniale, refusée par Henri III en 1259, était maintenant possible. En termes de rapport de forces, en revanche, il faut bien voir que l'initiative était venue du roi d'Angleterre : Erik Magnusson vivait surtout sur les acquis de 1281. L'ouverture politique vers l'Occident du roi norvégien s'était quelque peu émoussée. Mais le royaume occupait toujours, grâce aux liens familiaux tissés en 1281, une place importante sur l'échiquier international: il faut voir là le résultat de la politique matrimoniale menée depuis les années 1250.

Pour marier son fils avec Marguerite, Édouard I ${ }^{\text {er }}$ devait d'abord négocier avec le roi de Norvège. La délégation déjà évoquée arriva auprès d'Édouard $\mathrm{I}^{\mathrm{er}}$ en Gascogne avant le $1^{\mathrm{er}}$ mai $1289{ }^{45}$. En échange de son soutien à Marguerite, il dut certainement demander le mariage entre les deux enfants. Avant le 10 mai 1289, en effet, le souverain anglais, sans doute fort de l'accord des Norvégiens, envoya depuis la Gascogne une ambassade auprès du pape pour obtenir une dispense, afin qu'Édouard de Caernavon puisse épouser Marguerite, sa cousine au troisième degré ${ }^{46}$. Il restait maintenant à convaincre les Écossais, d'une part, de recevoir Marguerite comme reine et, d'autre part, d'accepter le mariage.

Rentré en Angleterre en août 1289, Édouard prolongea le sauf-conduit de la délégation norvégienne et convoqua les Écossais pour négocier. Le 6 novembre 1289, un accord fut conclu à Salisbury entre les Écossais, les Anglais et les Norvégiens: Marguerite était reçue comme reine par les Écossais, et devait arriver dans les îles Britanniques avant le $1^{\text {er }}$ novembre 1290, libre de tout mariage; les Écossais s'engageaient à pacifier leur royaume et à ne pas la marier sans l'accord du roi de Norvège et du roi d'Angleterre ${ }^{47}$. Le traité prévoyait une réunion entre Écossais et Anglais à la prochaine mi-carême pour confirmer et achever les discussions. Cette réunion avait pour but de convaincre les Écossais, sans aucun doute peu favorables à un mariage anglais et désireux de limiter les possibilités d'intervention d'Édouard dans les affaires internes du royaume, des avantages que pourrait entraîner une telle alliance. Les négociations aboutirent à la conclusion d'un traité de mariage entre les Écossais et les Anglais, à Brigham, le 18 juillet $1290^{48}$. Celui-ci prévoyait un gouvernement séparé des deux royaumes et leur transmission à deux héritiers distincts.

Les perspectives qu'offrait ce mariage étaient très intéressantes pour la Norvège : si jamais Håkon, le frère d'Erik de Norvège, venait à mourir, et en l'absence de collatéraux directs, un fils unique d'Édouard et de Marguerite pourrait prétendre à monter sur les trônes de Norvège, d'Angleterre et d'Écosse. Ce mariage, qui assurait définitivement la place de la dynastie norvégienne dans le vaste réseau familial des monarchies occidentales, n'a été possible que parce que la Norvège occupait une place suffisamment importante sur l'échiquier politique et stratégique occidental pour procurer au roi d'Angleterre ce qu'il désirait: une porte d'entrée en Écosse et, plus qu'une alliance, une garantie de non-agression de la part de l'Écosse et la Norvège dans le conflit qui se profilait avec la France.

35 Entre-temps, la dispense pontificale datée du 16 novembre 1289 était arrivée en Angleterre ${ }^{49}$. Édouard en avait informé le roi de Norvège et avait acheté l'arrivée rapide de Marguerite en Écosse, pour répondre aux exigences écossaises ${ }^{50}$. La future épousée arriva dans les Orcades, au nord de l'Écosse, au début du mois de septembre 1290 et une délégation anglo-écossaise partit à sa rencontre pour célébrer le mariage, mais Marguerite mourut à la fin du mois de septembre de maladie. Le lien qui unissait 
le roi norvégien aux familles d'Écosse et d'Angleterre, et au-delà à la plupart des familles régnantes d'Occident, était rompu, tout comme s'éloignait le rêve de l'union des trois royaumes. L'échec était total.

Épilogue

Le roi norvégien continua malgré tout à regarder vers l'Occident, mais sa situation ne lui permettait plus de mener une politique ambitieuse. L'objectif des Norvégiens, assaillis de difficultés financières et privés avec la disparition de Marguerite d'une combinaison politique de grande envergure, était désormais davantage de rentabiliser le traité de 1281 et ils revinrent à des ambitions matrimoniales plus modestes et plus locales. La mort de Marguerite ouvrait une nouvelle période de crise en Écosse : deux grandes familles se disputaient la succession, les Balliol d'un côté et les Bruce de l'autre. Quand s'ouvrit, en mai 1291, le procès de dévolution de la couronne d'Écosse, la Great Cause, Erik se déclara candidat au trône en tant que père de la reine défunte et envoya des émissaires en septembre 1292. Cette candidature, sans lendemain, avait pour but de sauver ce qui pouvait encore l'être du mariage de 1281, c'est-à-dire essentiellement les accords financiers. Erik réclamait ainsi, entre autres, le paiement des arriérés de la dot de sa femme, et une somme de 100000 livres qu'il estimait être due par les Écossais pour avoir refusé de reconnaître immédiatement Marguerite comme reine d'Écosse. Ce fut également l'occasion pour Erik de trouver une nouvelle épouse : Isabelle Bruce, fille de Robert Bruce le Jeune, lui-même fils de Robert Bruce l'Ancien, candidat au trône d'Écosse. On ne sait rien des négociations qui ont conduit à ce mariage, si ce n'est qu'un sauf-conduit fut attribué à Robert Bruce le Jeune et à sa fille pour se rendre en Norvège le 28 septembre $1292^{51}$, à un moment où l'issue du procès de dévolution ne faisait plus de mystère: la couronne d'Écosse allait être attribuée à John Balliol par Édouard I ${ }^{\text {er }}$ aux dépens de Robert Bruce l'Ancien. Ce fut officiel le 6 novembre $1292{ }^{52}$.

Il semble donc que ce mariage n'ait pas été le résultat d'un pari norvégien sur la victoire de Robert Bruce l'Ancien et il faut plutôt y voir une alliance avec un puissant aristocrate implanté sur la côte ouest de l'Écosse, dans une région qui, située face aux Hébrides, entretenait traditionnellement beaucoup de contacts avec les Scandinaves. Pourtant, même s'il avait été débouté, Robert Bruce possédait toujours un droit sur le trône d'Écosse en cas de disparition fortuite de John Balliol sans héritier. Il était donc possible qu'Isabelle, la reine de Norvège, vît un jour son frère devenir roi d'Écosse, voire qu'elle-même devînt reine. Ce mariage, conclu en 1293, était, comme celui de 1281, riche d'avenir même s'il ne permettait en aucune façon de nouer des liens de parenté entre les rois de Norvège et les autres souverains occidentaux. Robert, le frère d'Isabelle, devint en effet roi d'Écosse en 1306, à la suite d'un coup d'État. À défaut de nouer des liens de parenté avec les autres monarques occidentaux et de renforcer l'avancée de la Norvège au cœur de l'Occident chrétien, cette union "de proximité » consolidait le lien historique qui unissait l'Écosse à la Norvège.

L'échec des ambitions norvégiennes se poursuivit pendant toute la fin du XIII ${ }^{\mathrm{e}}$ siècle et le début $\mathrm{du}_{\mathrm{XIV}}^{\mathrm{e}}$ siècle : les rois de Norvège, tout en se repliant sur l'aire scandinavobaltique, continuèrent à rechercher des alliances matrimoniales en Occident mais, cette fois-ci, dans l'aristocratie et non dans les familles régnantes. Leur but était plutôt d'obtenir des avantages économiques et financiers que de gagner en prestige. Ce fut le cas par exemple de la tentative de Håkon Magnusson, le frère d'Erik, de se trouver une épouse dans l'aristocratie anglaise. Elle nous est connue par la réponse favorable que 
lui donna Édouard $\mathrm{I}^{\mathrm{er}}$ dans une lettre du 15 juin 1294. Le but de cette tentative était clairement d'amadouer le roi d'Angleterre afin qu'il intervînt en Écosse pour accorder les paiements prévus par le traité de 1281. Mais celui-ci n'avait plus le contrôle de la situation en Écosse et ce mariage n'aboutit donc pas. Un mariage français fut envisagé pour Håkon, afin de consolider le traité d'alliance, aux clauses financières très intéressantes, signé entre Erik, peut-être irrité par le peu d'empressement d'Édouard, et Philippe le Bel, le 22 octobre $1295^{53}$. Il échoua encore et la Norvège acheva de se replier totalement sur la péninsule scandinave et la mer Baltique.

Le mariage de 1290 devait être la consécration de la politique entreprise dans les années 1240 par Håkon Håkonsson. Celle-ci visait à entrer dans le réseau familial des souverains occidentaux afin de gagner une véritable légitimité au sein de la communauté qu'ils formaient. Pour cela, Håkon avait développé sa présence politique et stratégique sur l'échiquier européen, en exploitant l'héritage viking de son royaume, mais en prenant garde également à s'aligner sur les règles en usage en Occident. De cette façon, il obtint une première reconnaissance. Il multiplia ensuite les initiatives pour rendre effective son intégration dans l'ensemble occidental. Il rechercha en particulier des alliances matrimoniales permettant de servir ses objectifs : maintenir son avantage politique, et le renforcer par le réseau de parenté, élément essentiel des stratégies de la société politique médiévale. Ce fut sous le règne de Håkon que les rois de Norvège, profitant d'une conjoncture favorable, furent le plus dynamiques sur la scène internationale.

Bien que le mariage de 1281 ait fait entrer la dynastie norvégienne dans la parenté des rois d'Angleterre et, par conséquent, dans un réseau d'alliances couvrant l'Occident entier, et ait entraîné à la suite d'un heureux concours de circonstances le mariage de 1290, celui-ci avait été voulu avant tout par le roi d'Angleterre: les rois de Norvège n'avaient plus l'initiative. Cette union, qui aurait dû consolider le lien familial entre les rois de Norvège et les autres souverains occidentaux via le roi d'Angleterre, devait constituer l'apogée de la présence norvégienne en Occident. Mais la précarité même du projet, qui n'aboutit pas, marqua finalement le début de son déclin. À la mort de Marguerite, les rois de Norvège, criblés également de dettes, perdirent tout leur avantage, faute de s'être ménagé une solution de rechange. La légitimité qu'ils avaient gagnée en entrant dans la grande famille des souverains occidentaux n'eut pas le temps de s'enraciner et un processus irréversible s'enclencha: la position de la Norvège sur l'échiquier occidental s'affaiblit considérablement, ce qui rendit malaisée toute tentative nouvelle de contracter des mariages prestigieux et condamna la royauté norvégienne au repli, hormis en Écosse avec laquelle des contacts, par ailleurs traditionnels, se poursuivirent. On retrouve alors une adéquation entre le rayonnement du royaume de Norvège, qui ne fut plus que local, et sa politique matrimoniale, qui ne visa plus que ses voisins. Signe des temps, la fille unique de Håkon, Ingeborg, fut mariée à Erik, frère du roi de Suède Birger, en $13022^{54}$, ce qui entraîna par un concours de circonstances favorables l'union des deux royaumes scandinaves.

41 Cet exemple illustre bien les limites de l'exploitation politique du mariage princier au Moyen Âge. Le royaume de Norvège, malgré tous ses efforts, ne parvint pas au XIII siècle à s'intégrer pleinement à l'ensemble politique occidental par le biais de sa politique matrimoniale. Ce type de politique est fragile car il dépend surtout de deux facteurs difficiles à maîtriser : la vie des époux et de leur capacité à avoir des enfants. Dans ce domaine, le calcul est aléatoire et les crises fréquentes: le hasard ou une 
intervention malveillante - d'autant plus probable que l'enjeu politique est grand peuvent transformer un mariage dont on n'attendait rien politiquement en grande réussite, ou bien ruiner un projet solide, c'est ce que souligne l'exemple du mariage de 1281. Une union durable aurait-elle garanti à la Norvège une position sur l'échiquier occidental plus assurée - et pacifique ? Rien n'est moins sûr. Personne en effet ne pouvait prévoir en 1303 que le mariage d'Édouard II avec Isabelle de France, prévu dans le cadre d'un traité de paix, deviendrait une des justifications de la guerre de Cent Ans.

\section{NOTES}

1. G. W. S. Barrow, K. Helle, M. Prestwich et B. E. CRAWford, « Studies commemorative of the Anniversary of the Death of the Maid of Norway ", Scottish Historical Review, 69, 1990, p. 120-184.

2. N. BJøRGO, Ø. RIAN, A. KAARTVEDT, Selvstendighet og union fra middelalderen til 1905, Oslo, 1995 (Norsk utenrikspolitiks historie, I), p. 60-70.

3. Sur les Vikings en général et sur leurs relations avec l'Occident, outre les deux ouvrages classiques de L. MusSET, Les Peuples scandinaves au Moyen Âge, Paris, 1951 et Les Invasions : le second assaut contre l'Europe chrétienne, Paris, $2^{\mathrm{e}}$ édition, 1971, deux synthèses récentes sont à noter : The Cambridge History of Scandinavia, K. HeLle dir., Cambridge, 2003 et P. BAuduin, Les Vikings, Paris, 2004.

4. Sur le plan culturel, Håkon Håkonsson s'efforce de faire adopter en Norvège la culture courtoise qui triomphe en France et en Angleterre et est considérée comme moderne.

5. Notons que les mariages princiers avec les familles régnantes suédoises et danoises étaient courants depuis fort longtemps.

6. Titre non héréditaire, à l'exception du titre de jarl des Orcades, attribué par le roi de Norvège à un grand aristocrate qui se voit confier en même temps le gouvernement d'une partie du royaume. Ce titre fut réformé à partir de la fin du XIII e siècle. En 1308, il n'était plus réservé qu'aux membres de la famille royale et à l'aristocrate qui gouvernait l'archipel des Orcades, colonie norvégienne située sur la côte nord de l'Écosse.

7. Cette lutte est relatée dans la première partie de S. THORDARSON, Hákonar saga Hákonarsonar (HSH), G. VIGFUSSON éd., Londres, 1887 (Rerum Britannicarum medii aevi scriptores, LXXXVIII), aux chapitres 1 à 243. Voir aussi Norske konge sagaer, 4. Håkon Håkonssons saga, Oslo, 1979 (traduction en norvégien moderne).

8. K. HeLle, Og Norge blir en stat, 1130-1319, Oslo-Bergen-Troms $ø, 2^{\mathrm{e}}$ édition, 1974, p. 98-107.

9. N. BJøRGo, Ø. Rian, A. KAARTVEDT, op. cit., p. 70-77.

10. Il était le fils naturel et posthume du roi Håkon III.

11. Les registres de Grégoire IX (1227-1241), L. AUVRAY, S. VITTE-CLEMENCET, L. CAROLUS-BARRÉ éd., Paris, 1890-1955, vol. III, nº 6098 ; N. BJøRGO, Ø. RIAN, A. KAARTVEDT, op. cit., p. 70-77, p. 61-62. 
12. $H S H, \S 253-255$.

13. Entre 1220 et 1245 une trentaine d'ambassades au moins furent échangées entre l'Angleterre et la Norvège, selon les Regesta Norvegica, 822-1264, E. GUNNES éd., Oslo, 1989, vol. I.

14. Ibid., p. 63.

15. HSH, § 290 et 294. Cette histoire du règne de Håkon a été écrite en 1264-1265, peu de temps après la mort du grand roi en 1263. Pour plus de détails, voir notre article à paraître en 2006 dans Études Roussillonnaises, « Itinéraire d'une princesse norvégienne dans la péninsule Ibérique au XIII ${ }^{\mathrm{e}}$ siècle ».

16. $H S H, \S 284$.

17. $H S H, \S 287$.

18. Sur ces problèmes économiques, voir A. NEDKVITNE, Utenrikshandelen fra det vestfjelske Norge 1100-1600, Thèse de doctorat, Université de Bergen, 1983.

19. J. F. O'CALLAGHAN, The Learned King: the Reign of Alfonso X of Castille, Philadelphie, 1993, p. 201.

20. Ibid., p. 202.

21. N. BJøRgO, Ø. Rian, A. KaARTVEDT, op. cit., p. 64.

22. Diplomatarium Norvegicum (DN), t. XIX, A. BUGGE éd., Christiana, 1910-1914, nº 269 ; elle était incluse dans la réconciliation avec le duc de Bretagne et épousa Jean de Bretagne en 1260.

23. Par exemple, en 1277 , le code de la hird (hirdskraa) fut révisé et réorganisa les fonctions de l'entourage royal, qui était à l'origine la garde personnelle du roi et devint progressivement un organe de gouvernement; ses membres reçurent alors une titulature inspirée nettement de la féodalité occidentale : barons, chevaliers, seigneurs... Norges gamle love indtil 1387, R. KEYSER et P. A. MUNCH éd., Christiana, 1848. Hirdskraa, p. 391-450.

24. DN, t. VIII, C. C. A. LANGE, C. R. Unger éd., Christiana, 1871, $\mathrm{n}^{\circ} 9$.

25. DN, t. XIX, $\mathrm{n}^{\circ} 284$.

26. Certains passages de la Hákonar saga Hákonarsonar mettent en valeur le fait que les étrangers ont souvent une mauvaise opinion, injustifiée, du royaume de Norvège, entre autres au paragraphe 307. Cela apparaît également dans les chroniques écossaises qui n'épargnent pas en général le royaume de Norvège. Pour plus de détails, voir notre intervention « Redorer le blason : réécriture et légitimation dans la saga d'Håkon », à paraître dans les actes du colloque Le passé à l'épreuve du présent, tenu à l'université de Versailles-Saint Quentin du 27 au 29 janvier 2005.

27. Lui-même avait épousé Ingeborg Eriksdatter, fille du roi danois Erik Plovpenning.

28. Les proches du roi de Norvège, à l'image d'un Bjarne Erlingsson de Bjarkøy, exerçaient bien souvent une activité marchande lucrative, essentiellement avec les îles Britanniques; ils souhaitaient donc logiquement rester en bons termes avec l'Écosse qui était pour eux un passage obligé vers l'Angleterre et l'Irlande ( $D N, t$. XIX, $\mathrm{n}^{\circ} 456$ ). 29. Chronicon de Lanercost 1201-1346 (Chron. Lanercost), J. STEVENSON éd., Édimbourg, 1839, p. 104-105.

30. DN, t. XIX, no 305.

31. Cela permettait de régler le problème du paiement des arriérés liés au traité de Perth.

32. Né en 1268, Erik Magnusson était encore mineur lors de son avènement. Il succéda à son père sans difficulté : l'hérédité de la couronne était désormais bien établie. 
33. Chron. Lanercost, p. 104-105. Ce passage résume bien les préjugés négatifs qui courent sur le royaume de Norvège et leurs rois. La chronique précise en effet que Marguerite et son entourage étaient opposés à ce mariage car elle pouvait être mariée plus facilement et, nous citons, " plus convenablement » ailleurs qu'en Norvège.

34. Conformément au traité de Roxburgh, entre le 30 août et 8 septembre.

35. J. DE FoRdun, Johannis de Fordun Chronica Gentis Scotorum (Chron. Fordun), W. F. SKENE éd., Édimbourg, 1871-1872, chapitre 64.

36. Chron. Lanercost, p. 111.

37. La succession par voie féminine était tout à fait possible à cette époque en Écosse comme en Norvège mais par défaut, en l'absence d'héritier mâle ou de collatéral direct mâle. Ainsi, le frère du roi mort sans héritier mâle passait devant la fille unique du roi. C'est ce qui se passa en Norvège en 1299 : lorsque Erik mourut, il laissait une fille unique de son remariage avec Isabelle Bruce, c'est donc son frère Håkon qui lui succéda, en tant que plus proche successeur mâle du roi Magnus. Cette règle s'inspire de la règle de succession aux fiefs, à la différence que plusieurs héritières ne provoquent pas le partage du royaume comme c'est le cas pour le fief, c'est la plus âgée qui l'emporte.

38. DN, t. XIX, no 309.

39. Chron. Lanercost, p. 114 ; Chron. Fordun, chap. 67.

40. Chron. Lanercost, p. 115.

41. G. W. S. BARRow, "A kingdom in crisis : Scotland and the maid of Norway ", Scottish Historical Review, 69, 1990, p. 126.

42. Karlamagnus saga ok kappa hans, B. ViLHJÁLMSSON éd., Reykjavík, 1950, p. 50.

43. DN, t. XIX, $\mathrm{n}^{\circ} 328:[. .$.$] ad tractandum et conferendum cum serenitate vestra super$ quibusdam negociis, nos et filiam nostram karissimam Margaretam consanguineam vestram, dominam ac reginam Scotie, ac ipsum regnum suum Scotie tangentibus...

44. Ibid. : Excellenti et magnifico principi, affini et amico suo, domino Edwardo. Sur le vocabulaire de la parenté en latin médiéval, on peut se référer notamment à

A. GUERREAU-JALABERT, « La désignation des relations et des groupes de parenté en latin médiéval », Archivum Latinitatis Medii Aevi, 46, 1988, p. 65-108.

45. DN, t. XIX, $\mathrm{n}^{\circ} 330$.

46. Regesta Norvegica, 1264-1300, S. BAGGE, N. BJøRGo éd., Oslo, 1978, vol. II, nº 544.

47. DN, t. XIX, $\mathrm{n}^{\mathrm{o}} 333$.

48. $D N$, t. XIX, no 355 .

49. Les registres de Nicolas IV (1288-1292), E. LANGLois éd., Paris, 1905, nº 7526.

50. Lettre du 17 avril $1290 ; D N, t$. XIX, nº 346.

51. DN, t. XIX, $\mathrm{n}^{\circ} 379$.

52. DN, t. XIX, $\mathrm{n}^{\circ} 372$.

53. DN, t. V, C. R. UNGER éd., Christiana, 1860, n 29.

54. N. BJøRGO, Ø. RIAN, A. KAARTVEDT, op. cit., p. 92-93. 


\section{RÉSUMÉS}

Les rois de Norvège, à partir des années 1240, sous l'impulsion du roi Håkon Håkonsson, cherchent à intégrer durablement leur royaume, converti depuis à peine deux siècles, au sein de l'Occident chrétien. Entre autres stratégies, ils mettent en œuvre une politique matrimoniale visant à les faire entrer dans le vaste réseau familial des souverains occidentaux, et à profiter ainsi des systèmes d'alliances qui leur assureraient une place légitime dans le concert des royaumes occidentaux du XIII ${ }^{\mathrm{e}}$ siècle.

Les efforts des rois de Norvège pour s'aligner sur le modèle des royaumes d'Occident aboutirent à leur reconnaissance sur la scène occidentale en 1247. À partir de là, ils entreprirent une politique matrimoniale ambitieuse dont les effets restèrent néanmoins discrets : après une tentative sans lendemain vers la Castille, le mariage d'Erik de Norvège avec Marguerite d'Écosse en 1281 leur permit d'intégrer le réseau familial du roi d'Angleterre. Mais on retient surtout l'échec du projet de mariage de Marguerite, fille unique d'Erik et Marguerite, avec le fils aîné du roi d'Angleterre en 1290, qui aurait pu rendre durable l'intégration de la Norvège dans les combinaisons diplomatiques et politiques des monarchies occidentales. À la suite de cet échec, la Norvège se tourna davantage vers l'est et ses voisins scandinaves, ce qui entraîna à terme son union avec la Suède.

How to legitimate the integration into the Western Christendom : the wedding policy of the Kings of Norway in the thirteenth century. Our purpose is to show how the kings of Norway, from the $1240^{\text {ies }}$ on, in the wake of king Håkon Håkonsson, try to incorporate their newly converted kingdom into the Western Christendom. In this purpose, they build a wedding policy whose aim is to gain access to the western kings' large familial network, so that they can be considered as legitimate members of the xiIth century Western Christendom.

The kings of Norway's efforts to shape their kingdom according to western standards get them official recognition from the western community in 1247. From there on, they develop an ambitious wedding policy. Its greatest success is, although it is not commonly thus considered, in 1281, the wedding between King Erik of Norway and Marguerite of Scotland, thanks to which the kings of Norway become members of the king of England's familial network. But what is mostly remembered from this period is the failure in 1290 of the wedding between Margaret, Erik and Margaret's only daughter, and Edvard, the English king's first-born son. This wedding would have guaranteed Norway's long term integration into the diplomatic and political combinations of the western kingdoms. After this failure, the kings of Norway start focusing much more on their Scandinavian neighbours, which brings the union between Norway and Sweden.

\section{INDEX}

Mots-clés : politique matrimoniale, alliance, royauté, Norvège et Occident, négociations

\section{AUTEUR}

\section{FLORENT LENÈGRE}

Université Paris I Panthéon-Sorbonne, LAMOP, 17, rue de la Sorbonne, 75005 Paris Institut National du Patrimoine, 2, rue Vivienne, 75002 Paris 